\title{
Medical History Reported Term
}

National Cancer Institute

\section{Source}

National Cancer Institute. Medical History Reported Term. NCI Thesaurus. Code C83118.

The verbatim description of the medical history being reported. 